\title{
DISPERSAL IN COMPACT SEMIMODULES
}

\author{
ALEXANDER DONIPHAN WALLACE
}

(Received 24 November 1969)

Communicated by B. Mond

A semigroup is a nonvoid Hausdorff space together with a continuous associative operation. A semiring is a nonvoid Hausdorff space together with a couple of continuous associative operations, one of which (usually denoted as multiplication) distributes over the other (usually denoted as addition). If $R$ is a semiring then an $R$-semimodule is a semigroup $M$ under addition together with a continuous operation $R \times M \rightarrow M$ which satisfies the associativity and distributivity conditions usually stipulated in the instance of an $R$-module. It is purpose of this paper to establish for semimodules certain propositions proved by Kaplansky [4], Pearson [8], Selden [9], Beidleman-Cox [1] and others.

\section{1}

For results used here concerning semigroups reference is made to the excellent expository paper by Jane M. Day [2], the quite comprehensive dissertation by Paalman-de-Miranda [7] (though this omits algebraic topology) and the monumental Elements of Hofmann-Mostert [3]. The terminology is generally that common to these works.

If $(R,+, \cdot)$ is a semiring then the distributivity conditions are

$$
x(y+z)=x y+x z \text { and }(x+y) z=x z+y z,
$$

while those for a semimodule are

$\left(r r^{\prime}\right) m=r\left(r^{\prime} m\right), r\left(m+m^{\prime}\right)=r m+r m^{\prime}$ and $\left(r+r^{\prime}\right) m=r m+r^{\prime} m$.

Although none of their results will be used here, the reader may consult the numerous papers on semirings by Samuel Bourne, as well as a paper by Beidelman and Cox [1] on near-rings.

It should be explicitly stated that, in the definition of a semiring and a semimodule as used here, commutativity is nowhere demanded.

I am greatly indebted to K. R. Pearson for sending me a preprint of his paper [8], and to Mr. D. A. Robbie and Professor K. N. Sigmon for their kind comments and suggestions. Some of the results here will be given in more generality by $\mathrm{Mr}$. Robbie in his dissertation. 
The first result of this section extends propositions due to Kaplansky [4]. Pearson [8] and Beidleman-Cox [1]. The second extends another result of Kaplansky. While it would no doubt be possible to use local compactness and boundness, as Kaplansky did, attention is restricted here to compactness.

THEOREM 1. If $M$ is a compact additive group which is an R-semimodule over the compact semiring $(R,+, \cdot)$ with $(R,+)$ a group, and if $C$ is the component of $R$ containing the additive neutral element, then $C M=0$.

Before embarking on the proof it is desirable to state two propositions involving Lie groups, Montgomery-Zippin [5].

(A) A compact group is a Lie group if and only if there is some open set about the neutral element which properly contains no closed subgroup.

(B) If $G$ is a compact group and if $U$ is any open set about the neutral element then there is a morphism $f$ (continuous!) of $G$ onto a Lie group with $\operatorname{ker} f \subset U$.

Proof of Theorem 1 . Let $f$ be any morphism of $(M,+)$ onto the additively written Lie group $L$, let $W$ be an open set about the unit 1 of $L$ which properly contains no closed subgroup $(A)$, and define

$$
I(f)=\{r \mid f(r M)=1, r \in R\} .
$$

Notice that, as used here, a morphism is continuous and that $f\left(m+m^{\prime}\right)=$ $f(m) f\left(m j^{\prime}\right)$. It is readily concluded that $I(f)$ contains the neutral element 0 of $(R,+)$ and, indeed, that $I(f)$ is a subgroup of $(R,+)$. Since $0 M=0$, as is quickly verified, and since $M$ is compact and all operations are continuous by assumption. there is an open set $V$ about 0 such that $V M \subset f^{-1}(W)$, recalling that $f(0)=1$. When it is shown that $V \subset I(f)$, it may be concluded that $I(f)$ is an open (and hence closed) subgroup of $(R,+)$. Notice that whatever $r \in R$, the set $r M$ is a compact subgroup of $(M,+)$ and therefore $f(r M)$ is a closed subgroup of $L$. If $r \in V$ then $f(r M)$ is a subset of $W$ and a closed subgroup of $L$ and hence $f(r M)=1$. Thus, as indicated above, $I(f)$ is an open and closed subset of $R$ containing 0 , hence containing $C$, the component of $R$ which includes 0 .

To complete the proof, suppose that $\mathrm{cm} \neq 0$, with $c \in C$ and $m \in M$, and let $U$ in $(B)$ be $M \backslash\{\mathrm{cm}\}$ so that, with that morphism $f$, it devolves that $f(\mathrm{~cm}) \neq 1$, contrary to the conclusion of the preceding paragraph.

In the same fashion, mutatis mutandis, the following proposition may be established.

THEOREM 2. If $M$ is a compact additive group which is an R-semimodule over the compact semiring $(R,+, \cdot)$ with $(R,+)$ a group, and if $D$ is the component of $M$ which contains 0 then $R D=0$. 
An $R$-subgroup of the $R$-semimodule $M$ is such a subgroup $N$ of $M$ that $R N \subset N$.

The next proposition is readily proved.

LEMMA. If $N$ is an open (closed) subset of the compact $R$-semimodule $M$ over the compact semiring $R$ then

$$
J_{0}(N)=N \cap\{m \mid R m \subset N, m \in M\}
$$

is open (closed). If $N$ is a subgroup of $M$ then $J_{0}(N)$ is an $R$-subgroup of $M$, it being understood that $M$ is a group.

(C) It is convenient now to observe that any open set about the neutral element of a compact totally disconnected group includes a compact open subgroup, Montgomery-Zippin [5, 56].

THEOREM 3. If $M$ is a compact group which is an $R$-semi-module over the compact semiring $(R,+, \cdot)$ with $(R,+)$ a group, and if $M$ contains no closed $R-$ subgroups other than itself and 0 , then $M$ is finite.

Proof. If $D$ is the component of $M$ which contains 0 then $R D=0$, by Theorem 2, and since $D$ is a closed subgroup of $M$ it devolves that $D$ is a closed $R$-subgroup of $M$, and thus that $D=M$ or $D=0$.

On the first line, $M$ is connected and $R M=0$. If $M$ is a closed subgroup of then $N$ is also a closed $R$-submodule of $M$, thus there obtains the fact that $M$ properly contains no closed subgroup, and hence is a Lie group by (A). Accordingly $M$ contains (and therefore is) a toral group (cartesian product of circle groups) by Montgomery-Zippin [5, 190], whence $M$ itself is a circle group. Since the circle group properly contains closed subgroup it follows that $M$ has only one element.

On the second line, $M$ is totally disconnected, so that, by the lemma above and $(\mathrm{C})$, the complement of any member of $M$, other than 0 , contains an open and closed $R$-subgroup which must coincide with 0 , whence 0 is open. Accordingly, $M$ is seen to be finite.

\section{3}

The propositions of this section extend (and in one instance remedy a hiatus in) results of John Selden [9]. It is convenient to state first a result of Mycielski's [6].

(D) A compact group is connected if and only if it is divisible: if written additively, for each $x$ and each positive integer $n$ the equation ny $=x$ is solvable, by denoting the $n$-fold sum of $y$.

THEOREM 4. If $M$ is a compact $R$-semimodule over the compact semiring $R$ and if $T$ is an additive subgroup of $M$ such that $a T=T$ for some $a \in R$ then $T$ is totally disconnected. 
Proof. Since $a T^{*}=T^{*}$, since $T^{*}$ is a subgroup (because $T$ is, and because $M$ is compact) and if $T^{*}$ is totally disconnected the same will hold for $T$, it may and will be assumed that $T$ is closed. (For these arguments see Day [2] or Paalmande-Miranda [7].) By the Swelling Lemma, Day [2, 287], there is a multiplicative indempotent $e$ in $R$ such that $e T=T$, and if $C$ is the component of the neutral element of $T$ then $C$ is a compact connected group and, moreover, $e C=C$ because $e^{2}=e$. Indeed, $e x=x$ for any element $x$ of $T$. By (D) there is, for each $t \in C$ an element $t^{\prime}$ in $C$ such that the $n$-fold sum of $t^{\prime}$ is $t$ : otherwise, $C$ is divisible. From this it is concluded that

$$
(e+\cdots+e) C=C
$$

and, since

$$
\{t \mid t C=C\}
$$

is a closed set (see Day $[2,285]$ ) containing all $n$-fold sums of $e$, it contains the closure of the additive semigroup generated by $e$ thus an additive idempotent $f$, a result of Koch and Numakura, Day [2,285]. Whatever $t \in C$ there is some $t^{\prime} \in C$ with $t=f t^{\prime}$ and there obtains,

$$
t=f t^{\prime}=(f+f) t^{\prime}=f t^{\prime}+f t^{\prime}=t+t,
$$

thence $C$ is a group comprised of idempotents and therefore contains only one element. Whence $T$ is totally disconnected.

THEOREM 5. If $M$ is a compact $R$-semimodule over the compact semiring $R$, and if $m \in R m$ for each $m \in M$ then each additive subgroup of $M$ is totally disconnected.

Proof. By the Swelling Lemma cited in the proof of Theorem 4 it may be concluded, in virtue of the hypothesis $m \in R m$, that $m=e m$ for some multiplicative idempotent $e$ of $R$. Suppose that $T$ is an additive subgroup of $M$ with neutral element $z$, let $C$ be the component of $T$ which includes $z$, and let $t \in C$ distinct from $z$. There is a multiplicative idempotent $e$ of $R$ with $e t=t, e C$ is a subgroup of $M$ and satisfies $e \cdot e C=e C$. Because $e t \neq e z$ (otherwise $t$ would be an additive idempotent) the cardinal of $e C$ exceeds $1, e C$ is connected and is totally disconnected by Theorem 4 . This contradiction shows that $C$ is a singleton and thus that $T$ is totally disconnected.

While Theorem 5 does not subsume Selden's Theorem 1, such dependency can easily be obtained by generalizing the notion of a bimodule.

\section{References}

[1] Beidleman-Cox, 'Topological near-rings', Archive der Mathematik, 18 (1967), 485-492.

[2] Jane M. Day, 'Expository Lectures on Topological Semigroups', in M. A. Arbib (editor), Machines, Languages and Semigroups (Academic Press, New York, 1968). 
[3] Hofmann-Mostert, Elements of Compact Semigroups (Merrill, 1966).

[4] Irving Kaplansky, 'Topological rings', Amer. Journal Math. (19) 153-183.

[5] D. Montgomery and Zippin, Topological transformation groups, (Interscience, New York, 1955).

[6] Jan Mycielski, 'Some properties of connected compact groups', Colloquium Mathematicum 5 (1958), 162-166.

[7] A. B. Paalman-de Miranda, Topological Semigroups (Mathematisch Zentrum, Amsterdam, 1964).

[8] K. R. Pearson, The three kernels of a compact semiring, Journal Aus. Math. Soc. 10 (1969), 299-319.

[9] John Selden, 'A note on compact semirings', Proc. Amer. Math. Soc. 15 (1964), 882-886.

The University of Florida

Gainesville, Florida, U.S.A. 